\title{
Cidofovir Therapy for Adenovirus Pneumonia in an AIDS Patient on HAART: A Case Report
}

\author{
Trong Tony Trinh, Quy Ton, Robert Y. Choi
}

Division of Allergy and Infectious Diseases, Department of Medicine, University of Washington, Seattle, USA.

Email: tttrinh@u.washington.edu

Received September $12^{\text {th }}, 2012$; revised October $29^{\text {th }}, 2012$; accepted November $10^{\text {th }}, 2012$

\begin{abstract}
Introduction: Adenovirus infections are associated with significant morbidity and mortality among immunocompromised hosts. Adenovirus pneumonia is a rare and often fatal disease in patients with AIDS. Case Report: We report a case of a 28-year-old woman with advanced HIV/AIDS, who developed pneumonia four weeks after initiation of highly active antiretroviral therapy (HAART). Despite empiric antibiotics, the patient developed worsening hypoxemia and progressive pneumonia on chest x-ray. Culture data from a bronchoalveolar lavage (BAL) was negative for bacteria, fungi, pneumocystis jirovecii, but was positive for adenovirus detected by PCR. After transfer to a tertiary care hospital intensive care unit, a repeat BAL confirmed the presence of adenovirus by immunohistochemical staining. Tissue samples sent for histopathology revealed "smudge cells". Serum adenovirus viral load was $1.6 \times 10^{5}$ copies $/ \mathrm{mL}$. Intravenous cidofovir, $1 \mathrm{mg} / \mathrm{kg} /$ day, was initiated and scheduled three times a week. The patient exhibited remarkable improvement and was discharged to home in stable condition after four doses of cidofovir treatment. Discussion: Prior to our case, the few published accounts of HIV patients with adenovirus pneumonia treated with cidofovir have all resulted in death. Our case is distinct from these cases by the lack of concomitant pulmonary infection and the initiation of HAART prior to presentation. Conclusion: To our knowledge, we present the first case of adenovirus pneumonia in a patient with AIDS successfully treated with cidofovir. Our case suggests that limited and low dose cidofovir may be an efficacious approach to treat adenovirus pneumonia among HIV patients, especially those established on HAART.
\end{abstract}

Keywords: Adenovirus Pneumonia; AIDS; HIV; Cidofovir

\section{Introduction}

Adenoviruses are non-enveloped, double stranded DNA viruses of which over 50 serotypes have been identified. They are further classified into six subgroups (A to F) based upon immunologic, biologic and biochemical characteristics [1]. Adenoviruses have a worldwide distribution and infections occur throughout the year. Transmission occurs through aerosolized droplets, fecal-oral spread, contact with contaminated fomites or exposure to infected tissue or blood. Most adenoviruses readily infect epithelial cell lines and cause self-limited disease in patients with intact immune systems. Typical syndromes among the immunocompetent include fever, upper respiratory infections, conjunctivitis, urinary tract infections and diarrhea [2]. T-cell mediated and humoral immunity are integral in the control of and recovery from adenovirus infections [3]. Thus, adenovirus infections among immunocompromised hosts are associated with acute and persistent presentations, which lead to significant morbidity and mortality. In patients with HIV/AIDS, cases of hemorrhagic cystitis, prostatitis, nephritis, hepatitis, chole- cystitis, parotitis and encephatlitis have been reported [1]. Cases of adenovirus pneumonia are rare but usually fatal in patients with advanced HIV [1,4-6]. We present the first published case of adenovirus pneumonia in an AIDS patient on highly active antiretroviral therapy (HAART) treated successfully with IV cidfovir.

\section{Case Report}

In July of 2011, a 28 years old HIV-positive woman presented to a community hospital in Washington State with 5 days of progressive fever, dyspnea and non-productive cough. Since her HIV diagnosis in 2002, the patient endured a long history of opportunistic infections and nonadherence to HAART. Four weeks prior to presentation, her CD4 cell count was undetectable, her HIV-1 viral load was 131,700 copies/mL, and she was re-started on HAART (lamivudine/zidovudine, tenofovir, ritonavir boosted atazanavir). Three weeks prior, she was diagnosed with disseminated mycobacterium avium complex (MAC) and initiated treatment with azithromycin, ethambutol, and rifampin. On presentation, her vital signs 
were notable for a fever of $40.1^{\circ} \mathrm{C}\left(104.2^{\circ} \mathrm{F}\right)$, heart rate of 125 beats/minute, blood pressure of 119/60, an oxygen saturation of $91 \%$ on room air. Her labs showed a white blood cell (WBC) count of $1.5 \times 10^{3} / \mu \mathrm{L}$ (normal 2.1 - 10 $\mu \mathrm{L}$ ), hemoglobin of $9.5 \mathrm{~g} / \mathrm{dL}$ (normal $11.5-15.5 \mathrm{~g} / \mathrm{dL}$ ), platelets of $38 \times 10^{3} / \mu \mathrm{L}$ (normal $150-400 \mu \mathrm{L}$ ). Chest radiograph displayed an infiltrate in the right lower lobe and she was treated with supplemental oxygen and empiric broad-spectrum antibiotics (vancomycin and piperacillin/tazobactam) for healthcare associated pneumonia. Her HAART and MAC therapy were continued without interruption. A few days later, a bronchoscopy with broncho-alveolar lavage (BAL) was performed which was negative for acid fast bacilli, legionella, fungi, and pneumocystis jirovecii. Viral PCR from the BAL was positive for adenovirus, but treatment was not started. Over the next 7 days, her clinical status worsened, her oxygen demands increased and the decision was made to transfer her to a tertiary care center, Harborview Medical Center (HMC) in Seattle WA.

Upon transfer, her oxygen saturation was $85 \%$ on $5 \mathrm{~L}$ nasal cannula, $95 \%$ on a non-rebreather mask at $100 \%$ $\mathrm{FiO}_{2}$, and the patient was admitted to the intensive care unit (ICU). Her chest radiograph showed worsening consolidation in the right lower lobe, and her labs showed persistent pancytopenia. On HMC day 4, she underwent a repeat bronchoscopy. Fluid from the BAL was again found to be negative for fungi and bacteria, but the viral PCR revealed adenovirus. Tissue sent for histopathology revealed no significant inflammatory or granulomatous process but was notable for basophilic intranuclear inclusions resembling "smudge cells" (Figure 1). Immunohistochemical staining confirmed adenovirus (Figure 2). Serum adenovirus viral load by PCR was $1.6 \times$ $10^{5}$ copies/mL (Abbott m2000rt, Abbott Park, IL). The adenovirus was subsequently identrified to be subspecies B, serotype 21.

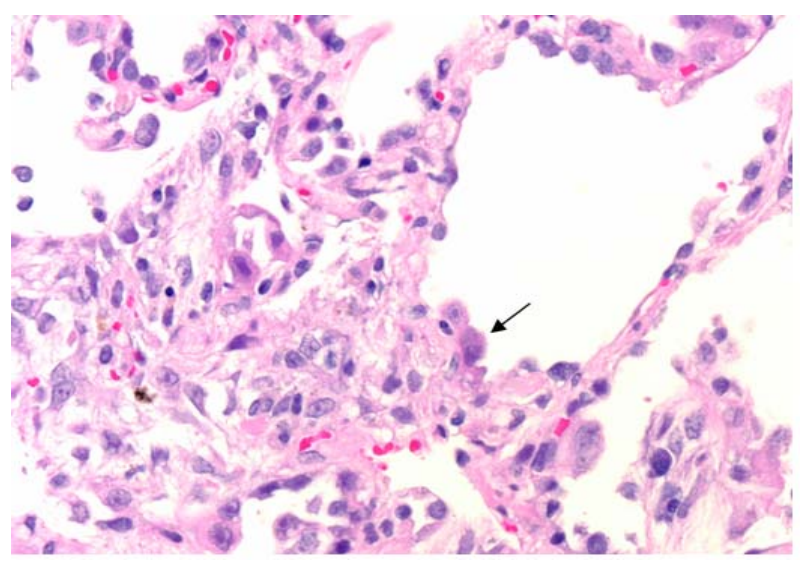

Figure 1. H \& E, 40×, Pneumocytes with enlarged nuclei and smudgy viral inclusions (arrow) protruding into alveolar spaces.
The decision was made to treat her adenovirus pneumonia with IV cidofovir at $1 \mathrm{mg} / \mathrm{kg} /$ day scheduled 3 times a week with aggressive IV hydration and prophylactic probenecid for renal protection. She was dosed on days $6,8,10$ and 12 and subsequently exhibited remarkable improvement. Her oxygen requirement decreased, and by day 13 oxygen saturation was $97 \%$ on room air. Her serum adenovirus declined to 58 copies $/ \mathrm{mL}$ and there was near radiographic resolution of her pulmonary infiltrate. Her CD4 count had increased to $8 / \mu \mathrm{L}$, and her HIV viral load was down to 91 copies $/ \mathrm{mL}$. She was discharged to home in stable condition on hospital day 15.

\section{Discussion}

With a wide range of clinical syndromes, adenovirus infections are common among immunocompromised patients and are associated with high morbidity and mortality [5]. In hematopoetic stem cell transplant (HSCT) recipients, the rates of adenovirus infection (with and without overt symptoms) have ranged from $3 \%$ to $47 \%$, with the lower rate observed when systematic screening was not performed [3]. In a study of 76 adult HSCT recipients with symptomatic adenovirus disease, mortality rate was noted to be $26 \%$, with higher rates among patients with pneumonia (73\%) [7].

In a prospective study of $63 \mathrm{HIV}$-positive patients, the risk of acquiring adenovirus at one year was noted to be $28 \%$ (18/63), all but one of whom had adenovirus detected in their feces [2]. Disseminated disease with multi-organ involvement is often noted with the most frequent sites being gastrointestinal [2] and urinary [3]. Adenovirus pneumonia is less often reported but a review of case reports of adenovirus pneumonia in HIV patients showed that 17/24 (75\%) cases resulted in death [1].

Our case illustrates multiple factors contributing to the

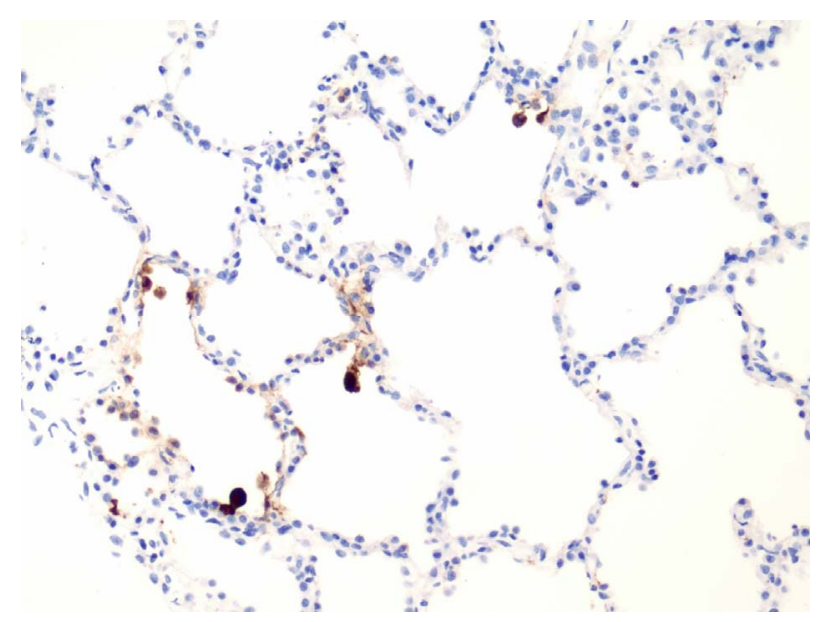

Figure 2. Adenovirus immunohistochemical Stain, 20×, Brown indicator highlights cells with adenovirus inclusions. 
complexity in diagnosing and treating adenovirus pneumonia in AIDS patients in the era of HAART. As was seen with disseminated MAC in our case, there is often a high rate of co-infection with other opportunistic pathogens. Given the timing of HAART to symptom onset, her undetectable pre-HAART CD4 cell count, and history of disseminated MAC, the immune reconstitution inflammatory syndrome (IRIS) was an early consideration in our patient. Systemic corticosteroids were considered but ultimately deferred as IRIS was deemed less likely when the pathology from the BAL revealed minimal inflammatory cells and adenovirus was identified. Furthermore, as it is a relatively rare diagnosis, many clinicians lack familiarity with adenovirus pneumonia leading to additional delays in recognition and appropriate therapy. As was demonstrated in our case, adenovirus was yielded early on, but therapy was not started until later in the patient's clinical course.

Cidofovir, ribavirin, and vidarabine are antiviral agents with reported efficacy against adenovirus. However, none of these agents have been studied in a prospective randomized controlled fashion. Cidofovir, a cytosine analog that inhibits viral DNA polymerase, appears to be the most effective and well documented. Cidofovir has been shown to be effective against all tested adenoviruses in vitro [8] and has been associated with successful outcomes in transplant recipients with adenovirus disease [9]. Common dosing regimens vary from $3 \mathrm{mg} / \mathrm{kg}$ to 5 $\mathrm{mg} / \mathrm{kg}$ weekly [10], to $1 \mathrm{mg} / \mathrm{kg}$ thrice weekly [11]. The most significant risk of cidofovir is dose dependent nephrotoxicity, which can be reduced with IV saline and probenecid. Probenecid, an organic acid, protects proximal renal tubular cells by preventing uptake of cidofovir into these cells, which has the paradoxical effect of decreasing renal clearance of the drug [12].

Although data is lacking on a standard cidofovir dosing regimen, we chose to implement the lower dosing range ( $1 \mathrm{mg} / \mathrm{kg}^{3}$ times a week) with aggressive IV hydration and probenecid co-administration to minimize nephrotoxicity. After cidofovir administration, our patient experienced dramatic clinical improvement with confirmed decrease in adenovirus viral load and radiographic resolution. Rapid clinical improvement in immunocompromised patients is not uncommon when adenovirus pneumonia is appropriately treated with antiviral therapy [13]. We used clinical response with documented viral suppression to dictate our treatment endpoint. Our patient only required four doses of cidofovir to achieve this.

Prior to our case, the very few published accounts of HIV patients with adenovirus pneumonia treated with cidofovir have all yielded poor outcomes. Nebbia and colleagues report a fatal case of disseminated adenovirus with worsening pneumonia and persistent viremia despite
IV cidofovir in addition to IV gancyclovir [6]. Adeyemi and colleagues similarly report a case of adenovirus pneumonia treated with ganciclovir and cidofovir that resulted in death [1]. Both of these cases were complicated by concomitant CMV pulmonary infection, which was not present in our case. Furthermore, the role of HAART in these cases was distinct from ours. In the former case, HAART was not initiated until late in the disease course. In the latter, HAART was not involved at all, before or during adenovirus treatment. In our case, our patient had already been initiated on HAART prior to her disease presentation.

Immune reconstitution, especially T-cell medicated immunity, is essential to successfully clearing adenovirus [11]. In vitro studies have shown that adenovirus elicits both CD4 and CD8 T-cell responses. In vitro removal of CD4 T cells (but not CD8 T cells) from a peripheral blood mononuclear cell population incubated with adenovirus removes an adenovirus-specific lymphoproliferative immune response [14]. This suggests that effective HAART is necessary to improve outcomes in AIDS patients infected with adenovirus. Our patient had been established on antiretroviral therapy approximately four weeks prior to her presentation and was continued on her regimen without interruption throughout her hospital course. By the time she was treated with cidofovir and clinically improved, she had completed approximately 8 weeks of antiretroviral therapy.

\section{Conclusion}

In summary, adenovirus pneumonia is a rare but significant cause of morbidity and mortality in immunocompromised patients and should be considered in those with advanced HIV and a refractory respiratory infection. Most published cases of adenovirus pneumonia in HIV patients result in death despite treatment. To our knowledge, we present the first published case of adenovirus pneumonia in a patient with AIDS successfully treated with cidofovir. Our case suggests that limited and low dose cidofovir may be an efficacious approach to treat adenovirus pneumonia among HIV patients, especially those established on HAART.

\section{REFERENCES}

[1] O. A. Adeyemi, A. V. Yeldandi and M. G. Ison, "Fatal Adenovirus Pneumonia in a Person with AIDS and Burkitt Lymphoma: A Case Report and Review of the Literature,” AIDS Reader, Vol. 18, 2008, pp. 196-198, 201-202, 206-207.

[2] S. H. Khoo, A. S. Bailey, J. C. de Jong and B. K. Mandal, "Adenovirus Infections in Human Immunodeficiency Virus-Positive Patients: Clinical Features and Molecular Epidemiology,” Journal of Infectious Diseases, Vol. 172, No. 3, 1995, pp. 629-637. doi:10.1093/infdis/172.3.629 
[3] M. Echavarria, "Adenoviruses in Immunocompromised Hosts,” Clinical Microbiology Reviews, Vol. 21, No. 4, 2008, pp. 704-715. doi:10.1128/CMR.00052-07

[4] H. Vinti, A. Pesce, B. Reboulot, E. Rosental, J. F. Michiels and J. P. Cassuto, "Fatal Adenovirus Pneumonia in an HIV-1 Infected Patient," IX International Conference on AIDS, 6-11 June 1993.

[5] J. Koopmann, F. Dombrowski, J. K. Rockstroh, U. Pfeifer, T. Sauerbruch and U. Spengler, "Fatal Pneumonia in an AIDS Patient Coinfected with Adenovirus and Pneumocystis Carinii,” Infection, Vol. 28, No. 5, 2000, pp. 323325. doi:10.1007/s150100070028

[6] G. Nebbia, A. Chawla, M. Schutten, C. Atkinson, M. Raza, M. Johnson and A. M. Geretti, "Adenovirus Viraemia and Dissemination Unresponsive to Antiviral Therapy in Advanced HIV-1 Infection," AIDS, Vol. 19, No. 12, 2005, pp. 1339-1340. doi:10.1097/01.aids.0000180115.26561.27

[7] A. M. La Rosa, R. E. Champlin, N. Mirza, J. Gajewski, S. Giralt, K. V. Rolston, I. Raad, K. Jacobson, D. Kontoyiannis, L. Elting and E. Whimbey, "Adenovirus Infections in Adult Recipients of Blood and Marrow Transplants," Clinical Infectious Diseases, Vol. 32, No. 6, 2001, pp. 871-876. doi:10.1086/319352

[8] F. Morfin, S. Dupuis-Girod, S. Mundweiler, D. Falcon, D. Carrington, P. Sedlacek, M. Bierings, P. Cetkovsky, A. Kroes, M. Van Tol and D. Thouvenot, "In Vitro Susceptibility of Adenovirus to Antiviral Drugs Is Species-Dependent,” Antiviral Therapy, Vol. 10, 2005, pp. 225-229.
[9] P. Ljungman, P. Ribaud, M. Eyrich, S. Matthes-Martin, H. Einsele, M. Bleakley, M. Machaczka, M. Bierings, A. Bosi, N. Gratecos and C. Cordonnier, "Cidofovir for Adenovirus Infections after Allogeneic Hematopoietic Stem Cell Transplantation: A Survey by the Infectious Diseases Working Party of the European Group for Blood and Marrow Transplantation,” Bone Marrow Transplant, Vol. 31, 2003, pp. 481-486. doi:10.1038/sj.bmt.1703798

[10] P. Ljungman, "Treatment of Adenovirus Infections in the Immunocompromised Host,” European Journal of Clinical Microbiology \& Infectious Diseases, Vol. 23, No. 8, 2004, pp. 583-588. doi:10.1007/s10096-004-1165-x

[11] C. A. Lindemans, A. M. Leen and J. J. Boelens, "How I Treat Adenovirus in Hematopoietic Stem Cell Transplant Recipients,” Blood, Vol. 116, No. 25, 2010, pp. 54765485. doi:10.1182/blood-2010-04-259291

[12] A. P. Lea and H. M. Bryson, “Cidofovir,” Drugs, Vol. 52, No. 2, 1996, pp. 225-231. doi:10.2165/00003495-199652020-00006

[13] C. Maslo, P. M. Girard, T. Urban, S. Guessant and W. Rozenbaum, "Ribavirin Therapy for Adenovirus Pneumonia in an AIDS Patient," American Journal of Respiratory and Critical Care Medicine, Vol. 156, 1997, pp. 1263-1264.

[14] P. Flomenberg, V. Piaskowski, R. L. Truitt and J. T. Casper, "Characterization of Human Proliferative T Cell Responses to Adenovirus,” Journal of Infectious Diseases, Vol. 171, No. 5, 1995, pp. 1090-1096. doi:10.1093/infdis/171.5.1090 